\title{
Heuristic Algorithm for Coordinating Smart Houses in MicroGrid.
}

\author{
Mohamed Arikiez $^{1}$, Floriana Grasso $^{1}$, and Michele Zito ${ }^{1}$ \\ ${ }^{1}$ Department of Computer Science, University of Liverpool, United \\ Kingdom
}

May 28, 2015

\begin{abstract}
This work presents a framework for efficiently managing the energy needs of a set of houses connected in a micro-grid configuration. The micro-grid consists of houses and local renewable plants, each seen as independent agents with their specific goals. In particular houses have the option to buy energy from the national grid or from the local renewable plants. We discuss a practical heuristic that leads to energy allocation schedules that are cost-effective for the individual houses and profitable for the local plants. We present experiments describing the benefits of our proposal. The results illustrate that houses and micro plants can make considerable saving when they work in micro grid compared with working alone.
\end{abstract}

\section{Introduction}

The world's energy needs are ever increasing $[1,2]$ and the investment in new power plants is not going to cover the future demand [3]. The power sector can be improved in many ways, using more renewable resources, or resorting to more efficient and environmentally friendly power plants. Also, Demand Side Management and Demand Response could encourage consumers to modify their energy usage behaviour.

The concept of Smart Grid is relatively new. The Smart Grid is an enhanced electrical grid in which information and communication technology is used to improve the power system and increase the profit of consumers, distributors and generation companies. The key features of such infrastructure are reliability, flexibility, efficiency, sustainability, peak curtailment, and demand response. The Smart Grid is also market enabling, it provides a platform for advanced services, and increases the manageability of the available resources. To exploit the Smart Grid in residential buildings, we need new technologies 
such as integrated communications, sensing and measurements, smart meters, advanced control, advanced components, power generation, and smart appliances [4]. Smart micro-grids [5] can be defined as a set of houses containing loads (appliances) and co-located resources (such as small PV arrays, or wind plants) working as a single controllable system [6]. Smart micro-grids also offer the possibility to export the surplus of locally generated power to the national grid.

There are plenty of studies that investigate methods for optimizing the cost of electricity in stand-alone residential buildings, based on electricity price, availability of renewable power, or user preferences. These studies use different algorithms to achieve their goals. For example, studies [7-10] use algorithms that find the optimal cost of electricity, whereas [11-13] use heuristic methods

which only guarantee suboptimal cost. Sharing local renewable power in small communities is an active research area. Study [14], uses Mixed Integer Linear Programming (MILP) to compare the cost of 20 houses working individually and the same houses working in a micro-grid setup. Although this study adds some knowledge to the field, it does not tackle the important issue of computation time. In studies of this type the time complexity of the particular algorithm increases with the system's granularity or the number of available appliances. The authors are only able to present examples that allocates resources over relatively large time slots. Furthermore, in this study each house uses only two appliances. Study [1] investigates the sharing of local renewable energy in a micro-grid. A greedy energy search algorithm is used to match the predicted renewable power with the predicted house consumption. The proposed approach also minimizes the power loss incurred while transferring electricity power along power lines by choosing the nearest house to share renewable power with. Unfortunately the proposed algorithm does not scale well with the length of the time slots.

In this work, we investigate the effectiveness of a MILP-based strategy that can be used to solve a particular energy allocation problem within a given microgrid. Our contribution is summarized in the following points:

- Design of a general micro-grid management system. Including fairly general notion of appliances.

- MILP applied to micro-grid control, with heuristic, for practical purposes.

- Preliminary empirical evaluation.

The rest of this paper is organized as follows: Section 2 details the system definition and modeling of system entities, MILP formulation is presented in section 3, and the fourth section illustrates the results which are followed by discussions in Section 5. Finally, the paper ends with conclusions.

\section{Allocation Problem}

In this section we present the formalization of the computational problem discussed in this paper. 


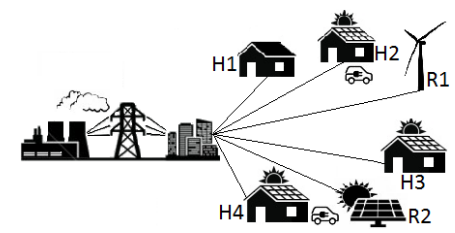

(a) Conventional Grid

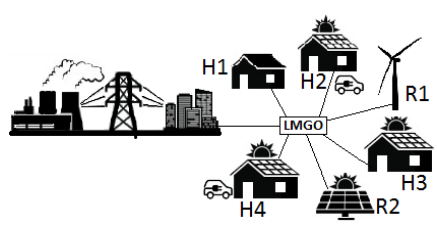

(b) Smart MicroGrid with LMGO

Fig. 1: Conventional grid vs smart MicroGrid

\subsection{The micro-grid}

A micro-grid consists of a set of houses $\mathcal{H}$, and a set of micro-generation power plants (or generators) $\mathcal{R}$ (see the example in Fig.(1)). Some houses (like H2, H3, or H4 in Fig.(1)) may be directly connected to a generator, and therefore they are able to receive energy from it in a particularly efficient way, but in general the houses in the system may receive their power from any of the generators in the micro-grid or the National Electricity Grid (NEG). The energy exchange within a micro-Grid is controlled by a Local micro-Grid Optimizer (LMGO). The power plants generate energy which can be either used by the houses in the micro-grid or exported to the NEG. Fig.(2) describes the possible energy exchanges between a house, a generator and the NEG. Houses (and their appliances) can only use electricity. The electricity comes in the house either from a generator (internal to the house or external) or from the grid. The labels on the arcs represent the unit cost that the entity at the end of the arrow will have to pay to the entity at the other end to get electricity from it. We assume that the energy produced by a generator $r$ can be sent to a house $h$ at a unit cost $\gamma_{r, h}$ or exported to the NEG at a cost $\zeta_{r}$. Alternatively, a house can buy energy from the NEG at a cost $\lambda_{h}$. All costs might change over time (hence the dependence on a time parameter $t$ shown in the figure).

\subsection{Appliances}

Each house $h \in \mathcal{H}$ is equipped with a set of appliances $\mathcal{A}_{h}=\left\{A_{1}, A_{2}, \ldots, A_{m_{h}}\right\}$, Appliances in a micro-grid are the main energy outlets. We assume that the appliances in the system can be easily switched on or off without disrupting

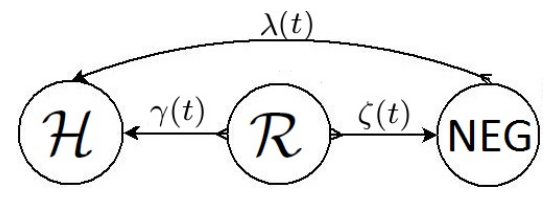

Fig. 2: Diagram shows exchange local renewable power among micro-grid components. 
their functionalities. Washing machines, cookers, air-conditioning (AC) units, battery chargers are examples of suitable appliances whereas TV sets or Computers do not fit into such framework. Moreover we assume that the appliances in a micro-grid can either be interruptible or uninterruptible, uniphase or multiphase. Interruptible appliances are designed to be switched $\mathrm{ON} / \mathrm{OFF}$ at any time. Appliances of this type include heaters, cookers, or air-conditioning (AC) units. Uninterruptible appliances are not designed to be switched OFF once they have been switched ON until they finish a particular task. Washing machines are good examples of uninterruptible appliances. Heaters are also examples of uniphase appliance. Any such appliance can either be OFF or ON and when it is ON it uses approximately a constant amount of power (nominal power). Note that the restriction to the use of uniphase appliances is that only have a single ON state, without loss of generality, appliances that can run at one of several power levels can be simulated by a combination of several uniphase appliances as defined in this paper. Multiphase appliances work in different phases, each using a certain amount of power. A washing machine typically uses a lot of energy at the beginning of a wash cycle, to heat up the water, then uses little energy for some time, and a bit more again during the final spins. Fig.(3.(b)) shows the typical power profile of three different multiphase appliances. Within a given phase, multiphase appliances cannot be switched off. We also assume that some appliances may have constraints on how often they are run while others might be controlled by environmental factors such as the level of charge of a battery, or particular desired values of room temperatures.

For the purpose of this study we assume that each appliance $A$ operates in $\Delta_{A}>0$ (nominal) phases and for each appliance, it is possible to define a power profile vector $\left(\alpha_{1}, \ldots, \alpha_{\Delta_{A}}\right)$ describing its energy needs. We assume that $\tau_{\min }$ is length of the shortest phase. Each $\alpha_{j}$ is a non-negative real number, corresponding to the average amount of power used by the appliance during its $j$ th phase. When switched on appliance $A$ progresses through each of its phases, starting from phase 1 up to phase $\Delta_{A}$ at which point the appliance is switched OFF. We also assume that for each appliance we know whether it is interruptible or not, and the number of times it must be used, $n_{A}$. Note that such model fits the different types of appliances described before.

\subsection{Optimization Problem}

From the discussion so far it is evident that a micro-grid consists of distinct agents each with their own goals and priorities: houses need energy to run their set of appliances according to pre-defined plans, generators produce energy that can be sold to the houses in the micro-grid or the NEG; houses want to purchase cheap energy whereas generators want to maximize their profit. In this setting we can associate a cost function $\Psi_{h}$ to each house $h \in \mathcal{H}$ :

$$
\Psi_{h}=\int \lambda_{h} L_{g}^{h} d t+\sum_{r} \int \gamma_{r}^{h} G_{r}^{h} d t
$$




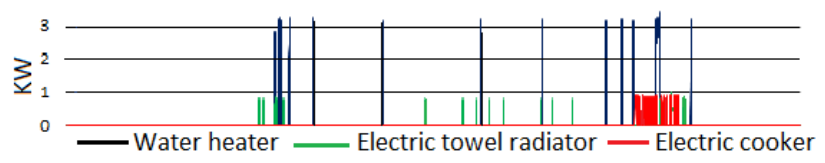

(a) Power profile of uniphase appliances

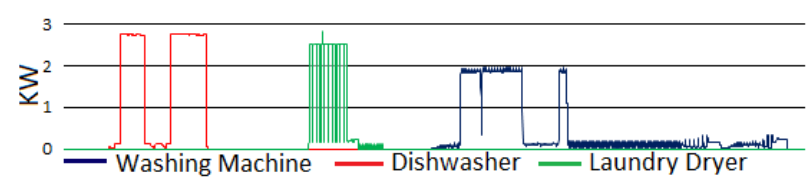

(b) Power profile of multiphase appliances

Fig. 3: Uniphase vs Multiphase appliances

where $L_{g}^{h}$ describes the amount of energy from the NEG used by house $h$ over time, and $G_{r}^{h}$ the amount of energy generated from plant $r$ used by $h$. Similarly, we can associate a profit function $\Xi_{r}$ to each $r \in \mathcal{R}$ :

$$
\Xi_{r}=\int \zeta_{r} E_{g}^{r} d t+\sum_{h} \int \gamma_{r}^{h} G_{r}^{h} d t
$$

In such formula, $E_{g}^{r}$ describes the amount of energy produced by $r$ that is sold to the NEG. If generator $r$ owned by house $h$ then $\Psi_{h}=\Psi_{h}-\Xi_{r}$. The problem of allocating energy to houses in a micro-grid in a way that is cost effective for the houses and profitable for the grid's power plants can then be cast as a multi-objective optimization problem [15].

$$
\min \left(\Psi_{h}: h \in \mathcal{H} ;-\Xi_{r}: r \in \mathcal{R}\right)
$$

In this paper we present a practical solution to this problem and provide some evidence of its effectiveness.

\section{MILP Formulation}

In this Section we present our heuristic algorithm for finding a feasible solution for the multi-objective optimization problem defined in Section 2.3. We start by providing a (multi-objective) mathematical programming formulation of the problem.

\subsection{Appliances modeling and linear constraints}

In what follows we assume that each instance of the problem is solved over a fixed time horizon (say 24 hours) and that time within such horizon is divided into a finite set of time slots, $\mathcal{T}=\left\{t_{1}, t_{2}, \ldots, t_{T}\right\}$, all of length $\tau$ with $0<\tau<\tau_{\min }$. We assume that $\tau$ divides the length of each phase within the system. We identify the $m_{h}$ appliances in house $h$ with the numbers $1,2, \ldots, m_{h}$. 


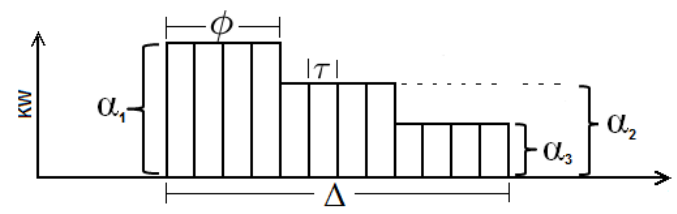

Fig. 4: Multiphase appliance modeling

Without loss of generality, we also assume that each appliance $i$ runs through $\Delta_{i}^{h}$ (real) phases, of length $\tau$. Note that real phases may be much shorter than the nominal phases mentioned in Section 2.2. Thus we assume that real phases are grouped into clutches corresponding to the nominal phases and the appliances are uninterruptible within each clutch (Fig.(4) shows an appliance with three clutches). We use a dedicated binary variable $x_{i, j}^{h}(t)$ for appliance $i$ in (real) phase $j$. The variable holds the appliance ON/OFF state at time $t$.

$$
P_{i, j}^{h}(t)=\alpha_{i, j}^{h} \cdot x_{i, j}^{h}(t) \in\left\{0, \ldots, \alpha_{\Delta_{i}^{h}}^{h}\right\} .
$$

We also assume that appliance $i$ in $h$ can only be run between time slot $t_{s}^{h, i}$ and $t_{f}^{h, i}$ (with $t_{s}^{h, i} \leq t_{f}^{h, i}$ ), in a so called comfort interval specified by the user, if needed. We model this using the following constraints

$$
\sum_{t=0}^{t_{s}^{h, i}-1} x_{j}^{h, i}(t)+\sum_{t=t_{f 1}^{h, i}+1}^{t_{T}} x_{j}^{h, i}(t)=0
$$

where either sums may be empty if $t_{s}^{h, i}=t_{1}$ or $t_{f}^{h, i}=t_{T}$. If both equalities hold (say if the user does not specify a comfort interval) the constraints vanish. To enforce that appliance $i$ in $h$ runs $n_{i}^{h}$ times in $\left\{t_{s}^{h, i}, \ldots, t_{f}^{h, i}\right\}$, we need the following constraints

$$
\sum_{t \in\left\{t_{s}^{h, i}, \ldots, t_{f}^{h, i}\right\}} x_{j}^{h, i}(t)=n_{i}^{h} .
$$

Phases can be kept in order imposing

$$
\sum_{t \in \mathcal{T}}\left[t \cdot x_{j+1}^{h, i}(t)-t \cdot x_{j}^{h, i}(t)\right] \geq 1 .
$$

and to prevent interruption between any two consecutive phases, we use constraint (7) with "=" replacing " $\geq$ ".

As mentioned before, the operation of some appliances depends on external conditions rather than initial user demands. For instance charging a battery depends on the battery charging state $\Theta_{i}^{h}(t)$ and its charging rate, $\alpha_{i}^{h}$, whereas the operations of an Air Conditioning (AC) unit depends on the room temperature, $T_{i n}^{h, i}(t)$, the outside temperature and the device heating or cooling power [8]. Appropriate constraints in such cases replace those in (6). In the case of batteries, say, we need to use the following constraints. 


$$
\begin{gathered}
\Theta_{i}^{h}(t)=\Theta_{i}^{h}(t-1)+\frac{1}{4} \cdot \pi \cdot P_{i, 1}^{h}(t) \quad \forall t: t \in\left\{t_{s}^{h, i}, \ldots, t_{f}^{h, i}\right\} \\
\Theta_{i}^{h}\left(t_{s}^{h, i}\right)=\underline{\beta}_{i}^{h}, \quad \Theta_{i}^{h}\left(t_{f}^{h, i}\right)=\bar{\beta}_{i}^{h}
\end{gathered}
$$

where $\underline{\beta}_{i}^{h}$ is the initial state of charge of the battery, $\bar{\beta}_{i}^{h}$ is the desired final state of charge of the battery (usually full), and $\pi$ is the battery charging efficiency. In the case of heating/cooling units, the main task of the given unit is to keep the room temperature within the comfort level $\left[T_{\text {min }}^{h, i}, T_{\text {max }}^{h, i}\right]$ during $b_{i}^{h}$ specified time intervals $I_{1}^{h}, \ldots, I_{b_{i}}^{h}$. The relationship between room temperature and the power allocated to the appliance is shown in Eq. (10).

$$
\begin{array}{r}
T_{i n}^{h, i}(t)=\epsilon \cdot T_{i n}^{h, i}(t-1)+(1-\epsilon)\left[T_{\text {out }}(t)-\frac{\eta}{\kappa} P_{i, 1}^{h}(t)\right] \\
T_{m i n}^{h, i} \leq T_{i n}^{h, i}(t) \leq T_{\text {max }}^{h, i} \forall t: t \in I_{1}^{h} \cup \ldots I_{b_{i}}^{h}
\end{array}
$$

where $\epsilon$ is the appliance inertia, $\eta$ is efficiency of the system (with $\eta>0$ for a heating appliance and $\eta<0$ in the case of cooling), $\kappa$ is the thermal conductivity, $T_{\text {out }}(t)$ is outside temperature at time $t$.

\subsection{Objective Function and Additional Constraints}

For the purpose of our experiments we simplify the general model presented in Section 2.3. The cost function in Eq.(1) is replaced by the linear function

$$
\Psi_{h}=\sum_{t \in \mathcal{T}}\left\{\lambda(t) L_{g}^{h}(t)+\sum_{r \in \mathcal{R}}\left[\gamma_{r}^{h}(t) G_{r}^{h}(t)\right]\right\} \forall h: h \in \mathcal{H},
$$

and similarly, the profit function in Eq.(2) is replaced by

$$
\Xi_{r}=\sum_{t \in \mathcal{T}}\left\{\zeta(t) E_{g}^{r}(t)+\sum_{h \in \mathcal{H}}\left[\gamma_{r}^{h}(t) G_{r}^{h}(t)\right]\right\} \forall r: r \in \mathcal{R} .
$$

Note that we are assuming that the cost of the energy from the NEG, $\lambda$, and the profit obtained selling energy to the grid, $\zeta$, may vary over time but are otherwise identical for all houses and generators in the system. Also if $r$ belongs to $h$ then $\gamma_{r}^{h}(t)=0 \forall t$, and $\Psi_{h}$ is the right-hand side of (11) minus $\Xi_{r}$.

Few constraints need to be added to the system. There are the renewable power constraints

$$
E_{g}^{r}(t)+\sum_{h \in \mathcal{H}} G_{r}^{h}(t)=P_{r}(t) \quad \forall t: t \in \mathcal{T}, \forall r: r \in \mathcal{R},
$$

where $P_{r}(t)$ is the renewable power generated by $r$, and power balance equations, enforcing that the allocated power at any time slot, $t$, must equal power demand at that time

$$
L_{g}^{h}(t)+\sum_{r \in \mathcal{R}} G_{r}^{h}(t)=\sum_{i \in \mathcal{A}_{h}} \sum_{j=0}^{\Delta_{i}^{h}} P_{j}^{h, i}(t), \quad \forall t: t \in \mathcal{T},
$$


The key idea is to reduce the multi-objective problem to a single objective one using "modified version" $\epsilon$-constraint method [16] in order to treat all entities equally, and then use a MILP solver to find a feasible allocation. To this purpose we consider the MILP obtained by using the constraints listed in 3.1 along with the following objective function

$$
\operatorname{Min}\left\{\sum_{i=1}^{|\mathcal{H}|} \Psi_{i}-\sum_{i=1}^{|\mathcal{R}|} \Xi_{i}\right\}
$$

and extra constraints

$$
\begin{array}{cc}
\Psi_{h} \leq \tilde{\Psi}_{h} & \forall h: h \in \mathcal{H} \\
\Xi_{r} \geq \tilde{\Xi}_{r} & \forall r: r \in \mathcal{R}
\end{array}
$$

where $\tilde{\Psi}_{h}$, and $\tilde{\Xi}_{r}$ are the optimal costs of the energy allocation problem for house $h$ and renewable plant $r$, considered as isolated units connected solely to the NEG.

\subsection{MILP-based Heuristic}

Let MinCost denote the version of our problem restricted to a single house, with $m$ uniphase appliances, to be allocated in one of two possible time slots. Also assume that the available renewable power is always $\frac{1}{2} \sum_{i=1}^{m} \alpha_{i}$, and the NEG electricity price is $\lambda>0$. A straightforward reduction from the PARTITION problem [17] shows that MinCosT is NP-hard. Therefore there is little hope that the MILP defined in the previous section might be solved quickly if the number of appliances is large. In our experiments we resort to an MILP-based heuristic algorithm to get a feasible solution in acceptable time. The basic idea is to use an off-the-shelf LP-solver to generate a feasible solution but without running the optimization process to completion. The LP-solver uses dual relaxation to find a lower bound on the optimum and stops as soon as the difference between the cost of the best feasible solution so far and the lower bound on the optimum becomes smaller than a predefined threshold. Also, we can put time limit or deadline to stop the algorithm.

\section{Empirical Evaluation}

All the experiments in this work have been done on a $\mathrm{PC}$ with an $\operatorname{Intel}(\mathrm{R})$ core(TM) i7-2600 CPU @ 3.4 GHZ, RAM is 16 GB, 64-bit Operating System (windows 7). In addition, Gurobi [18] has been used to solve LP and MILP problems, whereas the Java was the main tools to build our model. Three case studies will be demonstrated to illustrate the advantages and disadvantages of our approach. 


\subsection{First case study}

The main goals of this case study is to show the effect of renewable power demand on saving or profit.

\subsubsection{Input setting}

20 houses with variant renewable power generation capacities, see Table (1), and three independent renewable plants (PV array with maximum generation capacity $=5 \mathrm{KW} / \mathrm{H}$, two wind turbines, with $5 \mathrm{KW} / \mathrm{H}, 10 \mathrm{KW} / \mathrm{H}$ generation capacity, respectively) will be used to investigate the performance of our algorithm. The power profiles of uninterruptible appliances are shown in Table.(2), whereas the interruptible appliances are given in Table (3).

In addition, $\tau=5$ minutes, $T=288$ time slots, $\zeta=4.5 \mathrm{P} / \mathrm{KWH}, \xi=0.0$ $\mathrm{P} / \mathrm{KWH}, \gamma(t)=8.5 \mathrm{P} / \mathrm{KWH}, \pi=0.8$. Regarding AC's parameters, $\epsilon=0.96$, $\eta=30 \mathrm{KW} /{ }^{\circ} \mathrm{C}, \kappa=0.98, T_{\min }=18.0{ }^{\circ} \mathrm{C}, T_{\max }=22.0{ }^{\circ} \mathrm{C}$. Fig.(5) shows solar and wind power generated in Liverpool, UK $\left(53^{\circ} 24^{\prime} \mathrm{N} 2^{\circ} 59^{\prime} \mathrm{W}\right)$, using $3.5 \mathrm{KW} / \mathrm{H}$ $\mathrm{PV}$ array and $2 \mathrm{KW} / \mathrm{H}$ wind turbine. These data will be approximated and scaled up/down to model variant set of PV arrays and wind turbines.
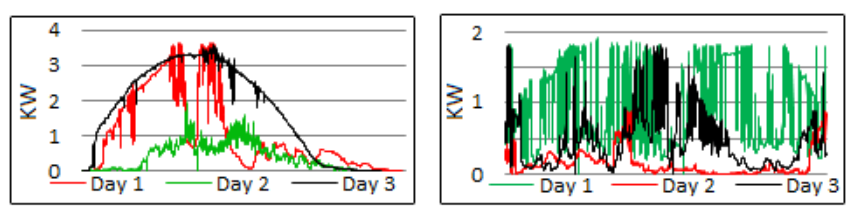

(a) Solar power for three days (b) Wind power for three days in in April, May, and June 2012 January, March, and June

Fig. 5: Renewable Power, for different three days in April, May and June 2012 in Liverpool

The electricity prices are shown in Fig.(6). In the first case study, dynamic pricing 1 will be used, whereas the rest will be used in the second and third case studies.

Table 1: PV array generation capacity of houses

\begin{tabular}{|c|c|c|c|c|c|}
\hline House No & $5,10,15$ & $1,6,11,16,19$ & $2,7,12,17,20$ & $3,8,13,18$ & $4,9,14$ \\
\hline \hline Capacity & $0.0 \mathrm{KW}$ & $1.0 \mathrm{KW}$ & $1.5 \mathrm{KW}$ & $2.0 \mathrm{KW}$ & $2.5 \mathrm{KW}$ \\
\hline
\end{tabular}




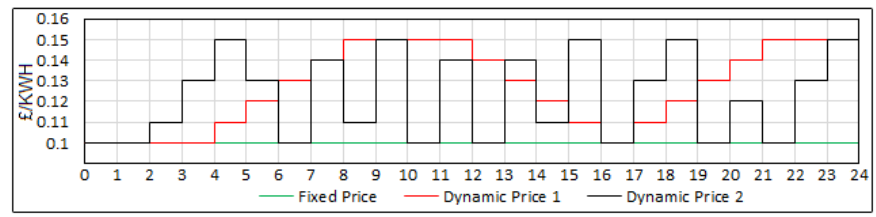

Fig. 6: Electricity Price, one fixed pricing scheme and two different dynamic pricing schemes.

Fig.(7) illustrates the outside temperature. Comfortable time for each appliance in each house is shown in Table(4). Three scenarios will be used in this case study to examine the effect of electricity demand on saving. These scenarios are low demand, medium demand, and high demand, See Table(5,6, and 7).

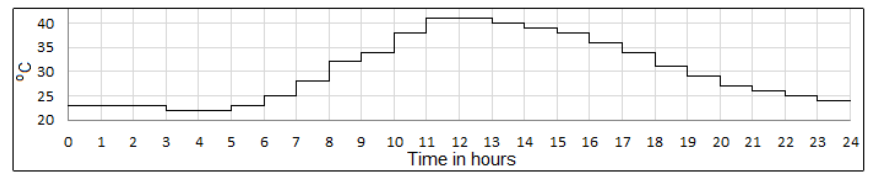

Fig. 7: Outside Temperature

\subsubsection{Findings}

Fig.(8a) displays the average profit of three scenarios, low demand, medium demand, and high demand. The houses with high demand, in general, can make more profit because the relationship between saving and renewable power consumption is positive. In contrast, $\operatorname{Fig}(8 \mathrm{~b})$ shows the relative MILP Gap of the three scenarios. As we can see, MILP Gap of high demand scenario is still above $100 \%$ after 30 minutes of running time that means the solution found could be far from optimality (we can save more by giving algorithm more time), it could be so close to optimality though. In addition, the first and second scenarios are so close to optimality because MILP gap is less than $1 \%$.

Table 2: Multiphase uninterruptible appliances

\begin{tabular}{|c|c|c|c|c|c|c|}
\hline Laundry Dryer & $\alpha$ in KW & 3.2 & 0.28 & 0 & 3.2 & 0.28 \\
\hline & $\phi$ in minutes & 15 & 10 & 5 & 20 & 10 \\
\hline Dishwasher & $\alpha$ in KW & 0.2 & 2.7 & 0.2 & 2.7 & 0.2 \\
\hline & $\phi$ in minutes & 5 & 15 & 15 & 20 & 5 \\
\hline Washing Machine & $\alpha$ in KW & 2.2 & 0.28 & 2.2 & 0.28 & - \\
\hline & $\phi$ in minutes & 10 & 20 & 10 & 20 & - \\
\hline
\end{tabular}




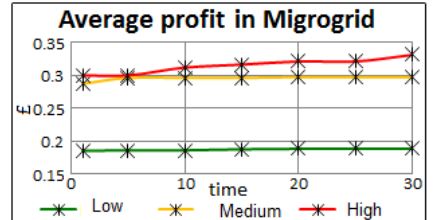

(a) Average profit in microgrid

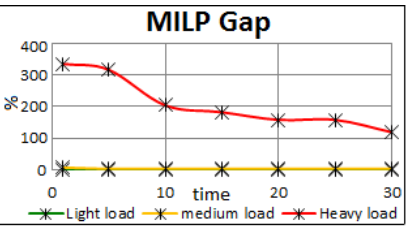

(b) the relative MILP Gap

Fig. 8: The result of low demand, medium demand, and high demand scenarios for 20 houses and 3 renewable independent plants

Fig. (9) depicts profit stability in low and high demand scenarios. The relationship between run time and average profit of all entities is positive, but it does not always hold for each entity. For instance, House No.17 in Fig.(9b) made more profit after 1,5 , and 10 minutes of calculation time than after 15 minutes but in general the average profit increases with time until it reach optimality. Fig.(10) illustrates the profit made by each component in micro-grid

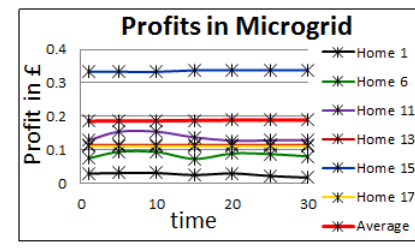

(a) Low demand

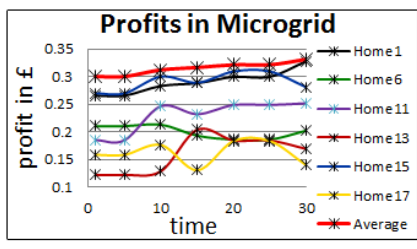

(b) High demand

Fig. 9: Profit stability in micro grid of first and third scenarios.

in three scenarios. Note that the first fife house in medium demand scenarios, surprisingly, made more profit than the first fife houses in high demand scenario because in medium demand scenario, the first fife houses has 6 to 7 appliances whereas the reset in around 4 and 5 houses so they consume a lot of local renewable power for cheap price, we have not put details about exact number of appliance and its details in each house for each scenarios due to page limit. Also, this behavior is expected in MILP heuristic algorithm because the solution is not optimal.

Table 3: Interruptible appliances

\begin{tabular}{|c|c|c|}
\hline Interruptible appliances & $\alpha$ & Depend on \\
\hline \hline Water heater & $3.1 \mathrm{KW} / \mathrm{t}$ & - \\
\hline Electric Towel Radiator & $1.5 \mathrm{KW} / \mathrm{t}$ & - \\
\hline Electric cooker & $2.5 \mathrm{KW} / \mathrm{t}$ & - \\
\hline Plug-in Hybrid Electric Vehicle & $0.35 \mathrm{KW} / \mathrm{t}$ & $\Theta\left(t_{s}\right)=2.0, \Theta\left(t_{f}\right)=16.0$ \\
\hline Air conditioner & $2.3 \mathrm{KW} / \mathrm{t}$ & $T_{\min }=18, T_{\max }=22$ \\
\hline
\end{tabular}


Table 4: Comfortable Interval

\begin{tabular}{|c|c|c|c|c|c|c|c|c|c|}
\hline House & Washing machine & Laundry dryer & Dishwasher & Water heater & Electric Radiator & \begin{tabular}{|l|} 
Electric cooker \\
\end{tabular} & PHEV & $\mathrm{AC}$, first period & $A C$, second period \\
\hline 1 & 10:00-13:00 & $13: 00-17: 00$ & 19:00-23:00 & 00:00-05:00 & 08:00-13:00 & 17:00-19:00 & $16: 00-23: 00$ & 13:00-15:00 & $17: 00-19: 00$ \\
\hline 2 & 9:00-12:00 & $12: 00-16: 00$ & 00:00-06:00 & 00:00-06:00 & 00:00-07:00 & $14: 00-17: 00$ & 00:00-06:00 & $13: 00-19: 00$ & $13: 00-19: 00$ \\
\hline 3 & $11: 00-14: 00$ & $14: 00-18: 00$ & $00: 00-07: 00$ & $10: 00-17: 00$ & $00: 00-05: 00$ & 17:00-19:00 & $00: 00-08: 00$ & $13: 00-19: 00$ & 13:00-19:00 \\
\hline 4 & 12:00-15:00 & 15:00-19:00 & 15:00-23:00 & 13:00-17:00 & 09:00-17:00 & 17:00-19:00 & 00:00-09:00 & 12:00-17:00 & 12:00-17:00 \\
\hline 5 & 8:00-11:00 & 11:00-15:00 & 14:00-19:00 & 02:00-07:00 & 10:00-13:00 & 16:00-18:00 & $00: 00-05: 00$ & 12:00-17:00 & 12:00-17:00 \\
\hline 6 & 9:00-12:00 & 12:00-16:00 & 19:00-23:00 & 10:00-16:00 & $17: 00-2300$ & 16:00-20:00 & 13:00-17:00 & 12:00-17:00 & 12:00-17:00 \\
\hline 7 & 10:00-13:00 & $13: 00-17: 00$ & 00:00-06:00 & 08:00-17:00 & $11: 00-16: 00$ & 18:00-20:00 & 09:00-20:00 & $10: 00-12: 00$ & 16:00-18:00 \\
\hline 8 & 8:00-11:00 & $11: 00-15: 00$ & $10: 00-15: 00$ & $16: 00-20: 00$ & 09:00-12:00 & $19: 00-22: 00$ & $11: 00-19: 00$ & $10: 00-12: 00$ & $16: 00-18: 00$ \\
\hline 9 & 10:00-13:00 & 13:0017:00 & 08:00-16:00 & 08:00-20:00 & 00:00-23:00 & 16:00-22:00 & 17:00-23:00 & 12:00-17:00 & 12:00-17:00 \\
\hline 10 & 10:00-13:00 & 13:00-17:00 & 19:00-23:00 & 00:00-05:00 & 08:00-13:00 & 17:00-19:00 & 16:00-23:00 & 13:00-15:00 & 17:00-19:00 \\
\hline 11 & 9:00-12:00 & 12:00-16:00 & 00:00-06:00 & 00:00-06:00 & 00:00-07:00 & 14:00-17:00 & 00:00-06:00 & 13:00-19:00 & 13:00-19:00 \\
\hline 12 & $11: 00-14: 00$ & $14: 00-18: 00$ & 00:00-07:00 & $10: 00-17: 00$ & 00:00-05:00 & $17: 00-19: 00$ & 00:00-08:00 & $13: 00-19: 00$ & $13: 00-19: 00$ \\
\hline 13 & $12: 00-15: 00$ & $15: 00-19: 00$ & $15: 00-23: 00$ & $13: 00-17: 00$ & 09:00-17:00 & $17: 00-19: 00$ & 00:00-09:00 & $12: 00-17: 00$ & $12: 00-17: 00$ \\
\hline 14 & 8:00-11:00 & 11:00-15:00 & 10:00-15:00 & 16:00-20:00 & 09:00-12:00 & 19:00-22:00 & 11:00-19:00 & 10:00-12:00 & 16:00-18:00 \\
\hline 15 & 9:00-12:00 & 12:00-16:00 & 00:00-06:00 & 00:00-06:00 & 00:00-07:00 & 14:00-17:00 & 00:00-06:00 & 13:00-19:00 & 13:00-19:00 \\
\hline 16 & 8:00-11:00 & 11:00-15:00 & 14:00-19:00 & 02:00-07:00 & 10:00-13:00 & 16:00-18:00 & $00: 00-05: 00$ & $12: 00-17: 00$ & 12:00-17:00 \\
\hline 17 & 8:00-11:00 & $11: 00-15: 00$ & $10: 00-15: 00$ & $16: 00-20: 00$ & $09: 00-12: 00$ & $19: 00-22: 00$ & $11: 00-19: 00$ & $10: 00-12: 00$ & $16: 00-18: 00$ \\
\hline 18 & 9:00-12:00 & 12:00-16:00 & 00:00-06:00 & 00:00-06:00 & $00: 00-07: 00$ & 14:00-17:00 & 00:00-06:00 & 13:00-19:00 & 13:00-19:00 \\
\hline 19 & 8:00-11:00 & 11:00-15:00 & 10:00-15:00 & 16:00-20:00 & 09:00-12:00 & 19:00-22:00 & 11:00-19:00 & 10:00-12:00 & 16:00-18:00 \\
\hline 20 & 8:00-11:00 & $11: 00-15: 00$ & 10:00-15:00 & $16: 00-20: 00$ & $09: 00-12: 00$ & 19:00-22:00 & $11: 00-19: 00$ & $10: 00-12: 00$ & $16: 00-18: 00$ \\
\hline
\end{tabular}

Table 5: the appliances in each house for low demand

\begin{tabular}{|c|c|c|c|c|c|c|c|c|}
\hline House & Washing machine & Laundry dryer & Dishwasher & Water heater & Electric Radiator & Electric cooker & PHEV & Air conditioner \\
\hline 1 & No & No & No & Yes & No & No & No & No \\
\hline 2 & Yes & No & Yes & No & No & No & No & No \\
\hline 3 & Yes & No & No & No & Yes & No & Yes & No \\
\hline 4 & No & Yes & No & Yes & No & Yes & Yes & No \\
\hline 5 & Yes & Yes & No & No & Yes & Yes & Yes & No \\
\hline 6 & Yes & No & Yes & Yes & Yes & Yes & Yes & No \\
\hline 7 & Yes & Yes & Yes & Yes & Yes & Yes & Yes & No \\
\hline 8 & Yes & Yes & Yes & Yes & Yes & Yes & Yes & Yes \\
\hline 9 & Yes & Yes & Yes & Yes & Yes & Yes & Yes & Yes \\
\hline 10 & Yes & Yes & Yes & Yes & Yes & Yes & Yes & Yes \\
\hline 11 & No & No & No & No & No & Yes & No & No \\
\hline 12 & No & No & Yes & No & No & No & No & No \\
\hline 13 & Yes & No & No & Yes & No & No & No & Yes \\
\hline 14 & No & Yes & No & Yes & Yes & No & No & No \\
\hline 15 & Yes & Yes & No & Yes & Yes & No & No & No \\
\hline 16 & Yes & No & No & No & No & Yes & No & Yes \\
\hline 17 & No & No & No & Yes & Yes & No & No & Yes \\
\hline 18 & Yes & No & Yes & No & No & Yes & No & No \\
\hline 19 & No & No & No & No & No & No & No & No \\
\hline 20 & No & No & No & No & No & No & No & No \\
\hline
\end{tabular}

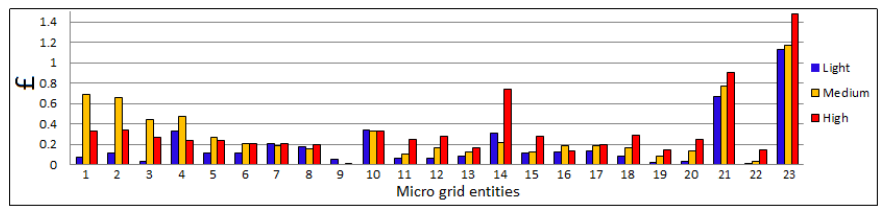

Fig. 10: The profit of entity in micro-grid in three scenarios.

\subsection{Second case study}

The main goal of this case study is to investigate the effect of electricity pricing on the profit. 
Table 6: the appliances in each house for medium demand

\begin{tabular}{|c|c|c|c|c|c|c|c|c|}
\hline House & Washing machine & Laundry dryer & Dishwasher & Water heater & Electric Radiator & Electric cooker & PHEV & Air conditioner \\
\hline 1 & Yes & Yes & Yes & Yes & Yes & Yes & Yes & NO \\
\hline 2 & Yes & Yes & Yes & Yes & Yes & Yes & Yes & NO \\
\hline 3 & Yes & Yes & Yes & Yes & Yes & Yes & Yes & NO \\
\hline 4 & Yes & Yes & Yes & Yes & Yes & Yes & Yes & NO \\
\hline 5 & Yes & Yes & Yes & Yes & Yes & Yes & Yes & Yes \\
\hline 6 & Yes & Yes & Yes & Yes & Yes & Yes & Yes & Yes \\
\hline 7 & Yes & Yes & Yes & Yes & Yes & Yes & Yes & Yes \\
\hline 8 & Yes & Yes & Yes & Yes & Yes & Yes & Yes & Yes \\
\hline 9 & Yes & Yes & Yes & Yes & Yes & Yes & Yes & Yes \\
\hline 10 & Yes & Yes & Yes & Yes & Yes & Yes & Yes & Yes \\
\hline 11 & No & No & No & No & No & Yes & NO & NO \\
\hline 12 & No & No & Yes & Yes & No & No & No & No \\
\hline 13 & Yes & No & No & Yes & No & No & NO & Yes \\
\hline 14 & Yes & No & No & No & No & Yes & NO & Yes \\
\hline 15 & Yes & Yes & No & Yes & Yes & No & NO & NO \\
\hline 16 & Yes & NO & NO & NO & NO & Yes & NO & YES \\
\hline 17 & NO & NO & NO & Yes & Yes & NO & NO & Yes \\
\hline 18 & Yes & NO & Yes & NO & NO & Yes & NO & NO \\
\hline 19 & NO & NO & NO & NO & NO & NO & NO & NO \\
\hline 20 & NO & NO & NO & NO & NO & NO & NO & NO \\
\hline
\end{tabular}

\subsubsection{Input settings}

We will use almost the same input data that have been used in third scenario in first case study but we will repeat the experiment with three pricing scheme, see $\operatorname{Fig}(6)$.

\subsubsection{Findings}

Fig.(11) shows the percentage profit made be each entity in micro grid with three pricing scheme. We used MIP gap $=25 \%$ to stop searching.

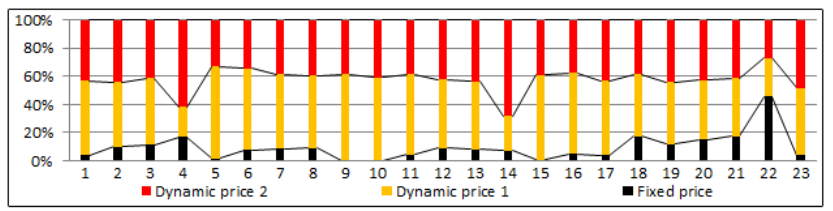

Fig. 11: The profits, in percentage, of micro grid when there is high demand (each house has 8 appliances) for fixed pricing scheme, and two different dynamic schemes.

The result illustrates that micro grid entities can make more profit in dynamic pricing scheme than in fixed pricing scheme. Note that entity no.22, surprisingly, made more profit in fixed pricing, that is because this solution is not optimal.

\subsection{Third case study}

The main goal of this case study is to examine the effect of number of houses in micro grid on the performance of our algorithm. Two scenarios will be demon- 
Table 7: the appliances in each house for high demand

\begin{tabular}{|c|c|c|c|c|c|c|c|c|}
\hline House & Washing machine & Laundry dryer & Dishwasher & Water heater & Electric Radiator & Electric cooker & PHEV & Air conditioner \\
\hline 1 & Yes & Yes & Yes & Yes & Yes & Yes & Yes & Yes \\
\hline 2 & Yes & Yes & Yes & Yes & Yes & Yes & Yes & Yes \\
\hline 3 & Yes & Yes & Yes & Yes & Yes & Yes & Yes & Yes \\
\hline 4 & Yes & Yes & Yes & Yes & Yes & Yes & Yes & Yes \\
\hline 5 & Yes & Yes & Yes & Yes & Yes & Yes & Yes & Yes \\
\hline 6 & Yes & Yes & Yes & Yes & Yes & Yes & Yes & Yes \\
\hline 7 & Yes & Yes & Yes & Yes & Yes & Yes & Yes & Yes \\
\hline 8 & Yes & Yes & Yes & Yes & Yes & Yes & Yes & Yes \\
\hline 9 & Yes & Yes & Yes & Yes & Yes & Yes & Yes & Yes \\
\hline 10 & Yes & Yes & Yes & Yes & Yes & Yes & Yes & Yes \\
\hline 11 & Yes & Yes & Yes & Yes & Yes & Yes & Yes & Yes \\
\hline 12 & Yes & Yes & Yes & Yes & Yes & Yes & Yes & Yes \\
\hline 13 & Yes & Yes & Yes & Yes & Yes & Yes & Yes & Yes \\
\hline 14 & Yes & Yes & Yes & Yes & Yes & Yes & Yes & Yes \\
\hline 15 & Yes & Yes & Yes & Yes & Yes & Yes & Yes & Yes \\
\hline 16 & Yes & Yes & Yes & Yes & Yes & Yes & Yes & Yes \\
\hline 17 & Yes & Yes & Yes & Yes & Yes & Yes & Yes & Yes \\
\hline 18 & Yes & Yes & Yes & Yes & Yes & Yes & Yes & Yes \\
\hline 19 & Yes & Yes & Yes & Yes & Yes & Yes & Yes & Yes \\
\hline 20 & Yes & Yes & Yes & Yes & Yes & Yes & Yes & Yes \\
\hline
\end{tabular}

strated.

\subsubsection{Input settings}

In the first scenario, we will use up to 20 identical houses (have the same power demand), each house has 8 different appliances, nominal power of appliances and comfortable time of each house are exactly the same in first case study. Each house equipped with PV array $(2.5 \mathrm{KW})$. There is no independent micro plant in this scenario.

In the second scenario, we will use up to 20 house and three independent micro plant in micro grid. We used the same data in third scenario in first case study. The only difference here is that we vary the number of houses from 1 to 20 .

\subsubsection{Findings}

Fig.(12) shows the average profit of houses, and the MILP gap in two scenarios. As we can see, the profit increases up to a point in both scenarios then it start decreasing with fluctuation that is because the calculation time is fixed ( 5 minutes) for all experiments, whereas the number of houses is varied. Moreover, micro grid with two houses will reach optimality or near-optimality faster than micro grid with 20 houses. In addition, it shows that the relationship between MILP Gap and number of houses is positive, and the relationship between MILP Gap and profit is negative. This explains why the curve of profit starts decreasing when MILP Gap curve start increasing. Black curves present the results of first scenario whereas the red curves present the results of second scenario. 


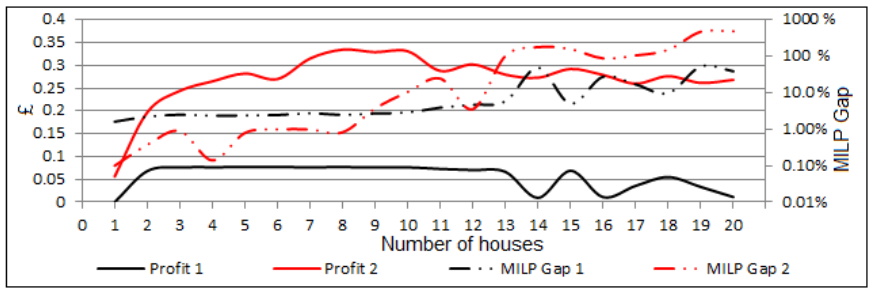

Fig. 12: Average cost and MILP Gap of houses in two scenarios

\section{Discussion and conclusion}

\subsection{Fairness issues}

By converting the problem from multi objectives to single objective one, fairness issue could raise. Constraints (16) and (17) are needed to reduce unfairness issue. Fig.(10) shows the individual profit of each entity. As we can see, house no. 5, 10, and 15, which are the houses that does not have PV array, made profit higher than house no. 9 (equipped with PV array). The main reason for this fairness issue is that this solution is suboptimal. Further work is needed to cope with this issue.

\subsection{Profit stability}

The stability of the profit depends on size of the problem (number of integer variables) and MILP Gap, if problem is small, the algorithm will reach optimality/near-optimality relatively fast and the profit will be almost stable, and vice versa. See Fig.(8) and Fig.(9).

Fig.(8b) shows, after 30 minutes of run time, the MILP gap still around $100 \%$, which means that the optimal solution may be still far on optimality, it could be so close, though.

\subsection{Scalability}

Increasing the number of entities in micro-grid does not always increase the profits of these entities. Fig.(12) shows that the relationship between the number of houses and profit is not always positive. As we can see in both scenarios, the profit has positive relationship with the number of houses up to a point, after that the relationship become negative, that is because we increase the number of houses whereas run time is fixed. Future work, this study provided preliminary investigation. Therefore, more investigation is needed to improve the fairness of the algorithm. Also, we can improve the efficiency of the micro-grid by prioritizing entities of micro grid.

To conclude, this work illustrates how an appropriate using MILP Heuristic can be for solving huge optimization problem, the results shows that the sub- 
optimal cost of each house in micro grid is cheaper than the optimal cost of each house working alone.

\section{References}

[1] Zhichuan Huang, Ting Zhu, Yu Gu, David Irwin, Aditya Mishra, and Prashant Shenoy. Minimizing electricity costs by sharing energy in sustainable microgrids. In Proceedings of the 1st ACM Conference on Embedded Systems for Energy-Efficient Buildings, pages 120-129. ACM, 2014.

[2] Anastasios I Dounis and Christos Caraiscos. Advanced control systems engineering for energy and comfort management in a building environment a review. Renewable and Sustainable Energy Reviews, 13(6):1246-1261, 2009 .

[3] Aldo Vieira Da Rosa. Fundamentals of renewable energy processes. Academic Press, 2012.

[4] Saima Aman, Yogesh Simmhan, and Viktor K Prasanna. Energy management systems: state of the art and emerging trends. Communications Magazine, IEEE, 51(1):114-119, 2013.

[5] Sunetra Chowdhury and Peter Crossley. Microgrids and active distribution networks. The Institution of Engineering and Technology, 2009.

[6] R.H. Lasseter. Microgrids. In Power Engineering Society Winter Meeting, 2002. IEEE, volume 1, pages 305-308 vol.1, 2002.

[7] Zhi Chen, Lei Wu, and Yong Fu. Real time price based demand response management for residential appliances via stochastic optimization and robust optimization. IEEE TRANSACTIONS ON SMART GRID, 2012.

[8] Tanguy Hubert and Santiago Grijalva. Modeling for residential electricity optimization in dynamic pricing environments. IEEE TRANSACTIONS ON SMART GRID, 2012.

[9] KM Tsui and SC Chan. Demand response optimization for smart home scheduling under real-time pricing. IEEE TRANSACTIONS ON SMART GRID, 2012.

[10] A Barbato and G Carpentieri. Model and algorithms for the real time management of residential electricity demand. In Energy Conference and Exhibition (ENERGYCON), 2012 IEEE International, pages 701-706. IEEE, 2012.

[11] E Matallanas, M Castillo-Cagigal, A Gutiérrez, F Monasterio-Huelin, E Caamaño-Martín, D Masa, and J Jiménez-Leube. Neural network controller for active demand-side management with pv energy in the residential sector. Applied Energy, 91(1):90-97, 2012. 
[12] Federica Mangiatordi, Emiliano Pallotti, Paolo Del Vecchio, and Fabio Leccese. Power consumption scheduling for residential buildings. In 2012 11th International Conference on Environment and Electrical Engineering (EEEIC), pages 926-930. IEEE, 2012.

[13] Thillainathan Logenthiran, Dipti Srinivasan, and Tan Zong Shun. Demand side management in smart grid using heuristic optimization. IEEE Transactions on Smart Grid, 3(3):1244-1252, 2012.

[14] Matthias Huber, Florian Sanger, and Thomas Hamacher. Coordinating smart homes in microgrids: A quantification of benefits. In 2013 4th IEEE/PES Innovative Smart Grid Technologies Europe (ISGT EUROPE), pages 1-5. IEEE, 2013.

[15] Pawel Malysz, Shahin Sirouspour, and Ali Emadi. Milp-based rolling horizon control for microgrids with battery storage. In Industrial Electronics Society, IECON 2013-39th Annual Conference of the IEEE, pages 20992104. IEEE, 2013.

[16] George Mavrotas. Effective implementation of the $\varepsilon$-constraint method in multi-objective mathematical programming problems. Applied Mathematics and Computation, 213(2):455-465, 2009.

[17] Alessandro Agnetis, Gianluca de Pascale, Paolo Detti, and Antonio Vicino. Load scheduling for household energy consumption optimization. IEEE Transactions on Smart Grid, 4(4):2364-2373, 2013.

[18] Inc. Gurobi Optimization. Gurobi optimizer reference manual, 2015. 